\title{
Should we be aghast at the AVAGAST data?
}

T

he use of antiangiogenic therapy as a treatment for solid malignancies has had a rocky road to travel in terms of approvals in the past year. After the recent recommendation for the withdrawal of FDA approval for the use of the VEGFtargeting antibody bevacizumab for the treatment of metastatic breast cancer, questions might have been asked about the utility of this therapy in other cancers. However, bevacizumab has demonstrated anticancer activity in some solid tumors, and remains approved for the treatment of several cancer types, including lung and colorectal cancer.

Roche, a company that distributes bevacizumab, has been pursuing the development of the therapy in other cancer types. One area of enquiry has been in patients with advanced-stage gastric cancer, and to that end they, in collaboration with clinicians, have conducted a randomized, doubleblind, placebo-controlled phase III trial (AVAGAST) to assess the use of bevacizumab in combination with chemotherapy in this patient group. The company explained that "the AVAGAST study was initiated on the basis of the broad activity of antiangiogenic inhibition in epithelial malignancies and encouraging results in a phase II study in metastatic gastric cancer."

The AVAGAST investigators recruited 774 patients with previously untreated, nonresectable, locally advanced or metastatic adenocarcinoma of the stomach or gastroesophageal junction. The patients were from centers in 17 countries; almost half were from the Asia-Pacific region (mostly Japan and Korea) with the rest from Europe (mostly Eastern Europe) and Pan-America (mostly Latin America).

The difference between this study and some other similar trials in other cancer types, such as colorectal cancer, was that the therapy was given in the first-line setting in advanced-stage disease, rather than later. The patients all received a chemotherapy regimen that consisted

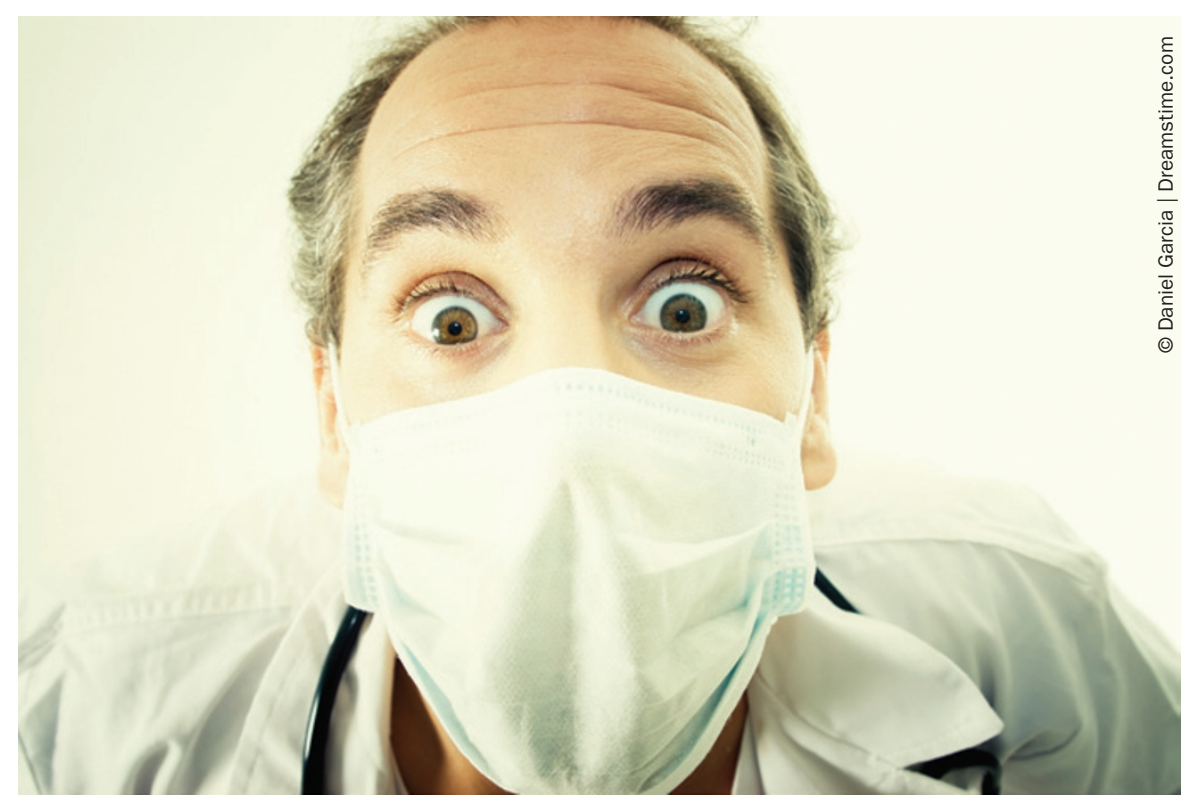

of fluoropyrimidine-cisplatin and were randomized in equal groups to receive either placebo or bevacizumab. The use of second-line treatment was equally distributed between the two arms, and only one patient in each arm received bevacizumab as a second-line therapy.

The primary end point of this trial was overall survival. Unfortunately, although there was a trend towards longer survival in the bevacizumab arm (12.1 months), this was not significantly different to the patients in the group receiving placebo (10.1 months). The data for one of the secondary end points-progressionfree survival-were more promising, with a significant improvement seen in the bevacizumab group compared with the placebo group (6.7 months versus 5.3 months). In addition, overall response rate was significantly improved in the bevacizumab group (46.0\% versus $37.4 \%$ ).

For Roche, the most important findings of the trial included that "subgroup analysis by geographic region indicated a survival benefit in the Pan-American region, whereas patients enrolled in Asia appeared to have no benefit and European patients had intermediate results.
Therefore, it is possible that improved (and significant) bevacizumab efficacy may be achieved by refining the selection of the patient population."

Bevacizumab has an established toxic-effect profile, and patients with advanced-stage gastric cancers have few treatment options, so it is possible that this geographical difference in therapy efficacy may offer hope for the further development of this therapy for some patients. This will require the establishment of biomarkers for response, which so far have been elusive for bevacizumab and other antiangiogenic therapies.

It seems that the jury is still out as to whether bevacizumab will become a firstline option for the treatment of patients with advanced-stage gastric cancer, and based on the data from trials of targeted therapies in other cancers, I would say this news is nothing to be aghast about!

\section{Rebecca Kirk}

Original article Ohtsu, A. et al. Bevacizumab in combination with chemotherapy as first-line therapy in advanced gastric cancer: a randomized doubleblind, placebo-controlled phase III study. J. Clin. Oncol. doi:10.1200/JC0.2011.36.2236 